\title{
A Distance-based Directional Broadcast Protocol for Urban Vehicular Ad Hoc Network
}

\author{
Da Li, Hongyu Huang, Xu Li, Minglu Li, Feilong Tang \\ Department of Computer Science and Engineering \\ Shanghai Jiao Tong University \\ Shanghai, China \\ \{leelion, sagittarius1131, colorfuldays_lee, mlli\}@sjtu.edu.cn, tang-fl@cs.sjtu.edu.cn
}

\begin{abstract}
The topology of a vehicular ad hoc network (VANET) changes rapidly due to high-speed movement of vehicles, so traditional mobile ad hoc network (MANET) broadcast protocol may not work efficiently in VANET. This paper proposes a distance-based broadcast protocol called Efficient Directional Broadcast (EDB) for VANET using directional antennas. In EDB, only the furthest receiver is responsible to forward the packet in the opposite direction where the packet arrives. Besides, a directional repeater located at the intersection helps disseminating the packets to the vehicles on other road segments of different directions. We evaluate the performance of EDB based on a real mobility model generated by live GPS data of taxis in the city of Shanghai. The result shows that EDB is effective and favorable for VANET.
\end{abstract}

Keywords-VANET; broadcast; directional antenna; mobility model; SUVnet

\section{INTRODUCTION}

In VANET, broadcast is a common means to disseminate messages such as emergency accident, traffic information services, announcements and advertisement. This kind of intervehicle communication improves traffic safety and travel comfort for drivers and passengers. Among various broadcast approaches, flooding is the first one. Each node rebroadcasts the received message exactly once, that results in broadcast storm problems [1]. Although [1] proposes mechanisms to improve flooding, they are not effective for all range of node density and packet loads in VANET. Due to the rapid change of topology, neighborknowledge based protocols [2] [3] [4] in MANET also do not work well in realistic scenario of VANET. Furthermore, crowded buildings which are built along the roads in the urban city make it difficult to transmit the packet to the different road segments separated by the buildings. In order to disseminate the message more efficiently, a suitable broadcast protocol should be designed for urban VANET.

Researchers in [5] design a broadcast protocol called Urban Multihop Broadcast (UMB) for VANET. The sender tries to select a far relay node in the intended direction on a linear road through request to broadcast (RTB) packet and clear to broadcast (CTB) packet. In order to disseminate the message in all directions, a repeater is employed at the intersection to rebroadcast the message. However, the protocol in [5] using omnidirectional antennas suffer from several drawbacks: redundant traffic, small coverage in sparse network, contention and collision especially at the intersection.
Since omnidirectional antennas bring in such drawbacks, many researchers study directional antennas to increase the network capacity and alleviate the interference [6] [7]. Compared with the omnidirectional antennas, directional antennas point to a certain direction with a small beamwidth that can achieve longer transmission range and better signal-to-noise ratio (SNR). Besides, when directional antenna is used for broadcast, it can alleviate the redundant transmission by forwarding the packet just in the direction where the packet does not arrive. Moreover, for some specific application in VANET, e.g. transmitting emergence brake message, the message only needs to be sent backward. However, researches of efficient broadcast protocol using directional antennas are limited. $\mathrm{Hu}$ et al. [8] advice three schemes to improve the broadcast efficiency using directional antennas in MANET. The basic idea is switching off the antenna to the known forward nodes. But it also leads to a large number of collisions caused by multiple receivers forwarding the message simultaneously in the same direction.

In this paper, we propose a novel broadcast protocol called Efficient Directional Broadcast (EDB) for urban VANET using directional antennas. EDB is composed of two parts: directional broadcast on the road and directional broadcast at the intersection. When a vehicle broadcasts on the road, only the furthest away receiver is responsible to forward the message just in the opposite direction where the packet arrives. At the intersection, a directional repeater is installed which is used to forward the message to vehicles on the different road segments incident to the intersection. Since the mobility models have critical impact on evaluation of broadcast protocols of VANET, a real mobility model is generated by mapping the GPS data of 4200 taxis onto a real road map of Shanghai. Based on this model we evaluate the performance of EDB with other two protocols. The results show EDB outperforms other broadcast protocols both in the delivery ratio and network resource consumption.

The rest of the paper is organized as follow: in section II we introduce the preliminaries on the real mobility model. Section III describes EDB protocol in detail. In Section IV we evaluate the performance of the proposed protocols with other protocols. Finally, we conclude in Section V.

\section{MOBILITY MODEL}

A practical urban vehicular network, which is called Shanghai Urban Vehicular Network (SUVnet), has been constructed in Shanghai, a metropolitan area. In preliminary stage of this work, we first collect data from 4200 taxis by GSM 
communication into a processing center. Such taxis are equipped with GPS devices which can provide longitude, latitude, timestamp, instant speed, Direction of Headway (DH), as well as the vehicle ID. Figure 1 gives a visualization of the SUVnet at the timestamp of 09:00:00 on 2006-11-22 where each black dot represents a taxi. The area of SUVnet is about $102 \mathrm{~km}^{2}$.

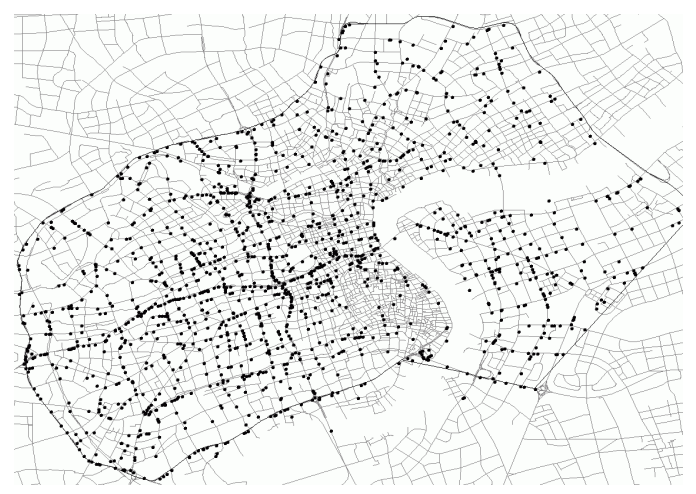

Figure 1. Visualization of SUVnet

In SUVnet, a taxi reports its position for about every 40 seconds and keeps silent in this interval. In order to generate the trace of taxi from the coarse-grain and imprecise data, we discuss three procedures used to pre-process the data for a better quality mobility trace. First, we match the GPS data onto the road map. Second, we identify a route between two consecutive data samples. Third, since the interval of two data samples is about 40 seconds, more points need be interpolated during this interval, e.g. every second.

The process of locating GPS data onto a road network map is called map-matching. In reality, due to various types of errors and imprecise information collected, the direct location based on its geographical location could lead to being mapped off the road, or on a wrong road. In order to improve in the accuracy of locating a vehicle onto a road map to help further data interpolation, we need to design a good map-matching algorithm to tolerate imprecise information with assistance of geometric matching mechanisms. The basic idea of our map-matching algorithm is described as follows: for a given position, we first filter the roads whose distance from this position is larger than 40 meters. Then we choose the one road with least value of distance and whose direction is not apart larger than 45 degrees from the $\mathrm{DH}$ of the position.

After the position of the vehicle is located, next we need to determine the route between any two consecutive sample data. An angle-based heuristic algorithm is developed, following the principles of minimizing turns. In SUVnet, the taxi keeps running on the current road until meets an intersection. If the next intersection along the same road is near to the destination than the current location, it will go straightly; otherwise it turns on another road which is nearer.
At last, by computing the time interval $t$ (second) and the length of trace distance $s$ (meter) of two consecutive data, intermittent data are generated for every $\mathrm{s} / \mathrm{t}$ meters.

\section{PROPOSED PROTOCOL}

In designing EDB, we make the following assumptions: (1) Each vehicle is aware of its geographical location by means of GPS device; (2) Since signals are obstructed by the crowed buildings on the roadsides in urban areas, packet can only be transmitted along the road; (3) Each vehicle is equipped with two fixed directional antennas with the beamwidth of 30 degree. These two antennas are mounted with one pointing front and the other pointing back which can be selected using a switch; (4)The vehicle listens in two directions when it is idle; (5) The MAC layer can learn the angle of arriver (AOA) and the strength of incoming signal power.

Under the above assumptions, the vehicle can disseminate the packet in one direction or both, by selecting the corresponding antennas. When receiving the packet, only the antenna which pointing to the sender is selected in order to decrease the interference from other direction.

\section{A. Directional broadcast on the road}

Due to the topology of VANET changed rapidly, EDB makes receiver-based decisions to forward the packet with the help of the GPS information. The receiver only needs to forward the packet in the opposite direction where the packet arrives. After a vehicle receives a packet successfully, it waits for a time before taking a decision whether to forward the packet or not. During this time, the vehicle listens to other relay of the same packet. The waiting time can be calculated using the following formula:

$$
\text { waitingTime }=\left(1-\frac{D}{T R}\right) * \max W T
$$

Where $\mathrm{D}$ is the distance from the sender which can be obtained using the sender's location information added in the packet and its own, and TR is the transmission range. The maxWT is a configurable parameter which can be adjusted according to the density of the vehicle.

From this formula, we can see the waiting time is proportion to the distance. So the waiting time of the furthest receiver expire first and it decides to forward the packet. In order to prevent other receivers relaying the same packet which will disturb the imminent forwarding, the furthest receiver transmits an ACK packet immediately towards the sender before forwarding. Thus, the other vehicles receiving this ACK packet are informed that they need not to forward the packet and finish waiting. Then, the packet is forwarded in the opposite direction where the packet arrives.

If the sender does not receive the ACK packet in maxWT, which indicates no vehicle forwards the packet, it will repeat the broadcast every $10 *$ maxWT until a next hop neighbor appears. 


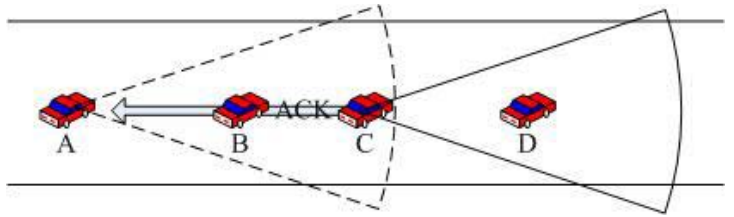

Figure 2. Directional broadcast on the road

To understand the basic principle of the protocol, consider a simple situation on the road in Figure 2. Vehicle A broadcast a packet rightward so both vehicle $\mathrm{B}$ and $\mathrm{C}$ receive the packet. After waiting for a distance-based time, vehicle $\mathrm{C}$ decides to forward the packet because it does not received any ACK packet during the waiting time which indicates no vehicle has relayed the packet. It immediately sends an ACK towards the vehicle A. So vehicle $B$ and A know that some other vehicle is responsible to forward the packet and finish waiting. At last $\mathrm{C}$ forwards the packet in the direction of vehicle $\mathrm{D}$, which is in the opposite direction where the packet arrives.

\section{B. Directional broadcast at the intersection}

In order to diffusing the packet to the vehicles on the other road segments, a directional repeater is installed at the intersection. The repeater is equipped with several directional antennas with the beamwidth of 30 degree pointing to the road segments incident to the intersection. These antennas can also be selected using a switch. Because the repeater has the best line-ofsight to the road segments, it is always forwarding the packet immediately to the different road segments after receiving the packet.

An example of intersection handling is shown in Figure 3. Repeater $\mathrm{R}$ is equipped with four fixed directional antennas pointing to four road segments. When $\mathrm{R}$ receives a packet from the vehicle $\mathrm{A}$, it at once forwards this packet in the directions of vehicle $B$ and $C$. In addition, the vehicle $D$ will receive the packet by the processing of directional broadcast on the straight road. Compared with the omnidirectional repeater used in [5] that needs to forward to each road segment respectively, the directional repeater reduces the traffic redundant and decrease the collision happen at the intersection.

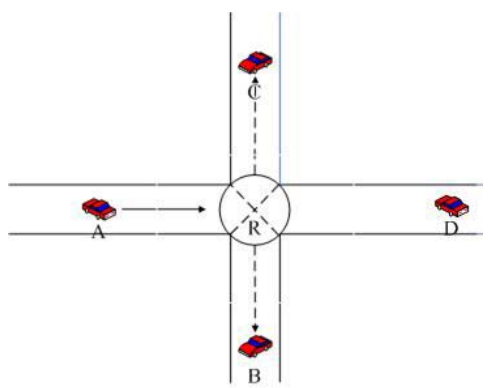

Figure 3. Intersection repeater
In [5], authors also address the loop problem which means the packet is sent back when there is a connected path consists of several intersections. As a result, the packet is transmitted on a road segment for multiple times and wastes bandwidth. In order to solve this problem, we propose that the repeater stores the received packet ID in a list for a certain time. It will check the list each times when a packet arrives. Every packet is forwarded only once.

\section{Performance Evaluation}

We develop an event driven simulator which models the network layer and MAC layer of wireless network. The channel is assumed to be error free. We evaluate the protocols based on the real mobility model SUVnet.

\section{A. Protocols}

In addition to EDB, We have simulated another two broadcast protocols with the same assumption of EDB. In this paper, we will refer to these protocols as Random Directional Broadcast (RDB) and Simple Distance-based Directional Broadcast (SDDB). In RDB, when the vehicle receives the broadcast packet, it waits for a random time before forwarding the packet in the opposite direction where the packet arrives. In SDDB, the receiver also waits for a Distance-based time calculated using the formula (1), but no ACK packet is used. All receivers in $\mathrm{RDB}$ and $\mathrm{SDDB}$ have to forward the packet. The parameter of maxWT in formula (1) is set to $10 \mathrm{~ms}$ and random waiting time for RDB is set to $10 \mathrm{~ms}$ too.

\section{B. Simulation Parameters}

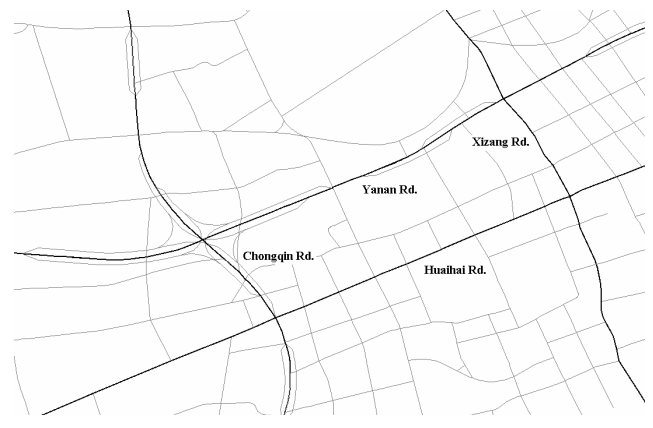

Figure 4. The road structure near People Square in SUVnet

Because the density of vehicle in SUVnet is various and the road structure is complex, we choose a type of road structure around People Square in Shanghai where the density of vehicle is high in Figure 4. The road structure contains four roads with four intersections. Each road is about $2.5 \mathrm{~km}$ long. There are about 90 vehicles moving on these roads, and the average vehicle density is about $9 \mathrm{veh} / \mathrm{km}$.

We assumed the packet length is 1024 bytes and the data rate is $1 \mathrm{Mbps}$. The directional gain for antenna is $10 \mathrm{~dB}$ and the 
transmission range using directional antenna is about 800 meters calculated using two-ray model which is also similar to [7]. The simulation lasts for 60 seconds.

\section{Results}

The simulator generates the packet at the rate of $10-50$ packets/second, and chooses a random vehicle as the source for each packet. The source broadcasts the packet in two directions simultaneously. Two metrics have been defined to compare the performance. (1) Delivery ratio: the ratio of the vehicles that receive a broadcast packet successfully to the total number of vehicles in the simulation. (2) Forward node ratio: the ratio of the forward vehicles to the whole vehicles for a given packet.

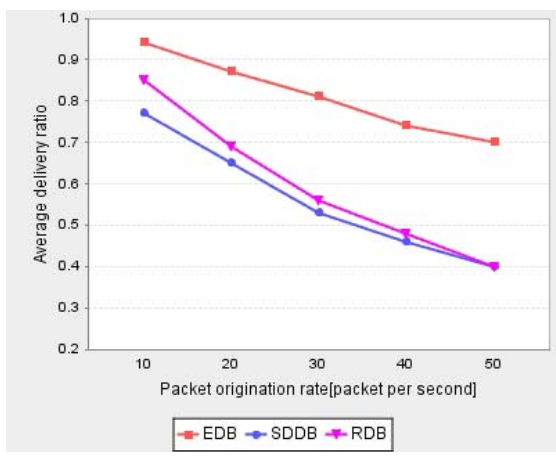

Figure 5. Average delivery ratio in SUVnet

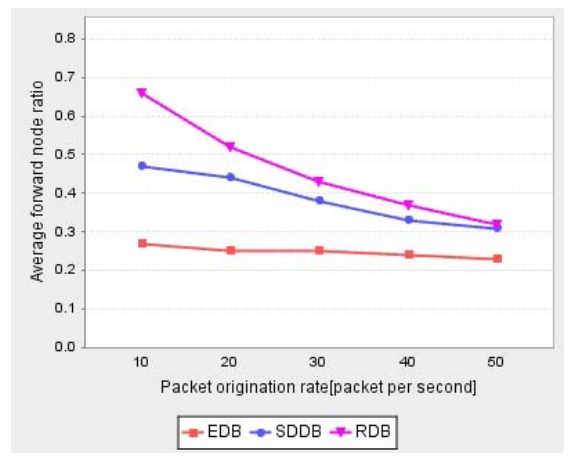

Figure 6. Average forward node ratio in SUVnet

As depicted in Figure 5, the average delivery ratio of EDB protocol outperforms the other two protocols. The reason is that in RDB and SDDB collision happened when multiple receivers forward the packet at same time. But in EDB only one vehicle is responsible to forward the packet using an ACK packet to notice other receivers. On the other hand, all three protocols suffer from the congestion of networks. As the packet origination rate increases, they begin to lose packets due to the contention and collisions.
In Figure 6, EDB also performs best in three protocols because only one vehicle is responsible to forward the packet for each broadcast. When the origination rate increases, the forward node ratio of $\mathrm{RDB}$ and $\mathrm{SDDB}$ begin to drops due to the lost packets. From these figures, we can conclude that EDB maximize the efficiency of relaying in delivery ratio and network resource consuming.

\section{CONCLUSION}

In this paper we present a distance-based broadcast called EDB for urban VANET. Since using directional antennas, EDB has too many advantages including long transmission range, space reuse, low redundancy and collisions. A real mobility model is generated by mapping the real-time GPS data of 4200 taxis onto a real road map of Shanghai. Based on this mobility model, we evaluate the performance of EDB and another two protocols. The result shows that EDB not only increases the delivery ratio but also decreases the network resource consumption. In our future work, we plan to improve the EDB to diffuse the message without any repeaters.

\section{ACKNOWLEDGMENT}

This paper was supported partially by National Natural Science Foundation of China, No. 60473092 and No. 90612018 , National Basic Research Program of China, No. 2006CB303000, 863 Program of China No. 2006AA01Z172, Natural Science Foundation of Shanghai Municipality of China No. 05ZR14081 and Intel Research Council.

\section{REFERENCES}

[1] S. Ni, Y. Tseng, Y. Chen, and J. Sheu. "The broadcast storm problem in a mobile ad hoc network.” Proc. MOBICOM'99, pp. 151-162, Aug. 1999.

[2] H. Lim and C. Kim. "Multicast tree construction and flooding in wireless ad hoc networks." In Proceedings of the ACM International Workshop on Modeling, Analysis and Simulation of Wireless and Mobile Systems (MSWIM), 2000.

[3] W. Peng and X. Lu. "On the reduction of broadcast redundancy in mobile ad hoc networks." In Proceedings of MOBIHOC, 2000.

[4] W. Lou and J. Wu, "On reducing broadcast redundancy in ad hoc wireless networks," IEEE Transactions on Mobile Computing, vol. 1, no. 2, pp. 111-123, Apr.-June 2002.

[5] G. Korkmaz,E. Ekici,F. Ozguner,and U. Ozguner, "Urban Multi-Hop Broadcast Protocols for Inter-Vehicle Communication Systems, ” in , Proc. of First ACM Workshop VANET 2004,pp. 76-85,Oct. 2004.

[6] T. Korakis, G. Jakllari, L. Tassiulas. "A MAC protocol for full exploitation of Directional Antennas in Ad-hoc Wireless Networks",in MobiHoc'03 June.

[7] R. R. Choudhury, X. Yang, N. H. Vaidya and R. Ramanathan, "Using directional antennas for medium access control in ad hoc networks," in Proc. ACM MobiCom, 2002.

[8] C. Hu, Y. Hong, and J. Hou. "On mitigating the broadcast storm problem with directional antennas.” In Proc. of IEEE ICC, May 2003. 